\title{
ASKEY-WILSON POLYNOMIALS AND A DOUBLE $q$-SERIES TRANSFORMATION FORMULA WITH TWELVE PARAMETERS
}

\author{
ZHI-GUO LIU
}

\begin{abstract}
The Askey-Wilson polynomials are the most general classical orthogonal polynomials that are known and the Nassrallah-Rahman integral is a very general extension of Euler's integral representation of the classical ${ }_{2} F_{1}$ function. Based on a $q$-series transformation formula and the Nassrallah-Rahman integral we prove a $q$-beta integral which has twelve parameters, with several other results, both classical and new, included as special cases. This $q$-beta integral also allows us to derive a curious double $q$-series transformation formula, which includes one formula of Al-Salam and Ismail as a special case.
\end{abstract}

\section{INTRODUCTION AND PRELIMINARIES}

Askey and Wilson [4] use the Askey-Wilson integral to find a set of orthogonal polynomials, which are now known as the Askey-Wilson polynomials. These polynomials are the most general classical orthogonal polynomials that are known. It is well-known that [10] the Askey-Wilson polynomials have five parameters, one of which is special, usually denoted by $q$, called a base. Ismail and Wilson [6] found a generating function for the Askey-Wilson polynomials. Szablowski [27] discussed some equivalent forms for the Askey-Wilson polynomials. Liu [17] found a new generating function of the Askey-Wilson polynomials, which was used to give a simple proof of the orthogonality for the Askey-Wilson polynomials.

It is generally known that it is a diffcult task to obtain double $q$-series transformation formulae. Al-Salem and Ismail [1] use $q$-Hermite polynomials to give an alternative evaluation of the Nassrallah-Rahman integral, and as a byproduct, they derive a double $q$-series transformation formula with six parameters. In this paper we will use the orthogonality relation for the Askey-Wilson polynomials to give a completely new proof of the Nassrallah-Rahman integral and then use this integral to prove a $q$-beta integral formula with 12 parameters which includes the Askey-Wilson integral as a special case.

This $q$-beta integral formula allows us to prove a double $q$-series transformation formula which contains the Al-Salem and Ismail double $q$-formula and some other double $q$-transformation formulas as special cases. We believe that our method is a new way of deriving $q$-formulas.

We now introduce some notations. For $0<q<1$, the $q$-shifted factorials are defined as

$$
(a ; q)_{0}=1, \quad(a ; q)_{n}=\prod_{k=0}^{n-1}\left(1-a q^{k}\right), \quad(a ; q)_{\infty}=\prod_{k=0}^{\infty}\left(1-a q^{k}\right) .
$$

Date: July 9, 2021.

The author was supported in part by the National Natural Science Foundation of China and Science and Technology Commission of Shanghai Municipality (Grant No. 13dz2260400).

2010 Mathematics Subject Classifications : 05A30, 33D15, 33D45, $11 \mathrm{E} 25$.

Keywords: Askey-Wilson integral, Askey-Wilson polynomials, Nassrallah-Rahman integral, $q$-beta integral. 
If $n$ is an integer or $\infty$ and $m$ is a positive integer, the multiple $q$-shifted factorial are defined as

$$
\left(a_{1}, a_{2}, \ldots, a_{m} ; q\right)_{n}=\left(a_{1} ; q\right)_{n}\left(a_{2} ; q\right)_{n} \ldots\left(a_{m} ; q\right)_{n} .
$$

As usual, the basic hypergeometric series or the $q$-hypergeometric series ${ }_{r+1} \phi_{r}$ is defined by

$$
{ }_{r+1} \phi_{r}\left(\begin{array}{c}
a_{1}, a_{2}, \ldots, a_{r+1} \\
b_{1}, b_{2}, \ldots, b_{r}
\end{array} ;, z\right)=\sum_{n=0}^{\infty} \frac{\left(a_{1}, a_{2}, \ldots, a_{r+1} ; q\right)_{n} z^{n}}{\left(q, b_{1}, b_{2}, \ldots, b_{r} ; q\right)_{n}} .
$$

The above $q$-hypergeometric series is called well-poised if the parameters satisfy the relations $q a_{1}=a_{2} b_{1}=a_{3} b_{2}=\ldots=a_{r+1} b_{r}$; very-well-poised if, in addition, $a_{2}=q \sqrt{a_{1}}$ and $a_{3}=$ $-q \sqrt{a_{1}}$.

For notational simplicity, we sometimes use the more compact notation

$$
{ }_{r+1} W_{r}\left(a_{1} ; a_{4}, a_{5}, \ldots, a_{r+1} ; q, z\right)
$$

to denote the very-well-poised series

$$
{ }_{r+1} \phi_{r}\left(\begin{array}{c}
a_{1}, q \sqrt{a_{1}},-q \sqrt{a_{1}}, \ldots, a_{r+1} \\
\sqrt{a_{1}},-\sqrt{a_{1}}, q a_{1} / a_{4} \ldots, q a_{1} / a_{r+1}
\end{array} ; q, z\right) .
$$

For $x=\cos \theta$, the Askey-Wilson polynomials $p_{n}(\cos \theta ; a, b, c, d \mid q)$ are defined as [4], [5, p. $188]$

$$
(a b, a c, a d ; q)_{n} a^{-n}{ }_{4} \phi_{3}\left(\begin{array}{c}
q^{-n}, a b c d q^{n-1}, a e^{-i \theta}, a e^{i \theta} \\
a b, a c, a d
\end{array} ; q, q\right) .
$$

Definition 1.1. For $x=\cos \theta$, we define $h(x ; a \mid q)$ and $h\left(x ; a_{1}, \ldots, a_{m} \mid q\right)$ as

$$
\begin{gathered}
h(x ; a \mid q)=\left(a e^{i \theta}, a e^{-i \theta} ; q\right)_{\infty}=\prod_{k=0}^{\infty}\left(1-2 q^{k} a x+q^{2 k} a^{2}\right), \\
h\left(x ; a_{1}, \ldots, a_{m} \mid q\right)=h\left(x ; a_{1} \mid q\right) h\left(x ; a_{2} \mid q\right) \cdots h\left(x ; a_{m} \mid q\right) .
\end{gathered}
$$

For notational simplicity, we will use $W_{(a, b, c, d)}(\cos \theta \mid q)$ to denote

or

$$
\frac{h(\cos 2 \theta ; 1 \mid q)}{h(\cos \theta ; a, b, c, d \mid q)}
$$

$$
\frac{\left(e^{2 i \theta}, e^{-2 i \theta} ; q\right)_{\infty}}{\left(a e^{i \theta}, a e^{-i \theta}, b e^{i \theta}, b e^{-i \theta}, c e^{i \theta}, c e^{-i \theta}, d e^{i \theta}, d e^{-i \theta} ; q\right)_{\infty}} .
$$

Proposition 1.2. For $\max \{|a|,|b|,|c|,|d|\}<1$, the Askey-Wilson q-beta integral formula states that

$$
\int_{0}^{\pi} W_{(a, b, c, d)}(\cos \theta \mid q) d \theta=K(a, b, c, d \mid q),
$$

where here, and throughout the paper, $K(a, b, c, d \mid q)$ is given by

$$
K(a, b, c, d \mid q)=\frac{2 \pi(a b c d ; q)_{\infty}}{(q, a b, a c, a d, b c, b d, c d ; q)_{\infty}} .
$$

There are several different ways of proving the Askey-Wilson $q$-beta integral formula, see, for example [3, 4, 7, 11, 13, 14, 20, 23, 25].

The orthogonality relation for the Askey-Wilson polynomials is stated in the following theorem (see, for example [10, p.384] and [12, p.416]). 
Theorem 1.3. Let $p_{n}(\cos \theta ; a, b, c, d \mid q)$ be the Askey-Wilson polynomials and $\delta_{m, n}$ the Kronecker delta. Then, for $\max \{|a|,|b|,|c|,|d|\}<1$, we have

$$
\begin{aligned}
& \int_{0}^{\pi} W_{(a, b, c, d)}(\cos \theta \mid q) p_{n}(\cos \theta ; a, b, c, d \mid q) p_{m}(\cos \theta ; a, b, c, d \mid q) d \theta \\
& =K(a, b, c, d \mid q) \frac{(1-a b c d / q)(q, a b, a c, a d, b c, b d, c d ; q)_{n}}{\left(1-a b c d q^{2 n-1}\right)(a b c d / q ; q)_{n}} \delta_{m, n}
\end{aligned}
$$

When $m=n=0$ the orthogonality relation for the Askey-Wilson polynomials reduces to the Askey-Wilson integral.

The Rogers ${ }_{6} \phi_{5}$ summation formula (see, for example [5, p.36]) is stated in the following proposition.

Proposition 1.4. For $|q \alpha / b c d|<1$, we have the q-summation formula

$$
{ }_{6} W_{5}\left(\alpha ; b, c, d ; q, \frac{q \alpha}{b c d}\right)=\frac{(q \alpha, \alpha q / b c, \alpha q / b d, \alpha q / c d ; q)_{\infty}}{(\alpha q / b, \alpha q / c, \alpha q / d, \alpha q / b c d ; q)_{\infty}} .
$$

We proved the following extension of the Rogers summation by using $q$-exponential differential operator in [15, Theorem 3]. For an alternative proof, see [16, Theorem 6.2].

Theorem 1.5. For $\max \left\{\left|\alpha \beta a b c / q^{2}\right|,\left|\alpha \gamma a b c / q^{2}\right|\right\}<1$, we have the identity

$$
\begin{gathered}
\sum_{n=0}^{\infty} \frac{\left(1-\alpha q^{2 n}\right)(\alpha, q / a, q / b, q / c ; q)_{n}}{(q, \alpha a, \alpha b, \alpha c ; q)_{n}}\left(\frac{\alpha a b c}{q^{2}}\right)^{n} \\
\times{ }_{4} \phi_{3}\left(\begin{array}{c}
q^{-n}, \alpha q^{n}, \beta, \gamma \\
q / a, q / b, \alpha \beta \gamma a b / q
\end{array} ; q, q\right) \\
=\frac{\left(\alpha, \alpha a c / q, \alpha b c / q, \alpha \beta a b / q, \alpha \gamma a b / q, \alpha \beta \gamma a b c / q^{2} ; q\right)_{\infty}}{\left(\alpha a, \alpha b, \alpha c, \alpha \beta a b c / q^{2}, \alpha \gamma a b c / q^{2}, \alpha \beta \gamma a b / q ; q\right)_{\infty}} .
\end{gathered}
$$

When $\gamma=0$, this theorem reduces to the $q$-summation formula due to Ismail- RahmanSuslov [8].

Upon replacing $c$ by $q t$ and then $(a, b)$ by $(q / a b, q / a c)$ in the equation above, we find that

$$
\begin{aligned}
& \sum_{n=0}^{\infty} \frac{\left(1-\alpha q^{2 n}\right)\left(\alpha, a b, a c, t^{-1} ; q\right)_{n}}{(1-\alpha)(q, q \alpha / a b, q \alpha / a c, \alpha t q ; q)_{n}}\left(\frac{\alpha t q}{a^{2} b c}\right)^{n} \\
& \quad \times{ }_{4} \phi_{3}\left(\begin{array}{c}
q^{-n}, \alpha q^{n}, \beta, \gamma \\
a b, a c, q \alpha \beta \gamma / a^{2} b c
\end{array} ; q, q\right) \\
& =\frac{\left(q \alpha, q \alpha t / a b, q \alpha t / a c, q \alpha \beta / a^{2} b c, q \alpha \gamma / a^{2} b c, q \alpha \beta \gamma t / a^{2} b c ; q\right)_{\infty}}{\left(q \alpha / a b, q \alpha / a c, q \alpha t, q \alpha \beta t / a^{2} b c, q \alpha \gamma t / a^{2} b c, q \alpha \beta \gamma / a^{2} b c ; q\right)_{\infty}} .
\end{aligned}
$$

The following proposition [15, Theorem 4] can be derived from the above equation by replacing $(\alpha, \beta, \gamma)$ with $\left(a b c d / q, a e^{i \theta}, a e^{-i \theta}\right)$.

Proposition 1.6. For $|d t|<1$, the Askey-Wilson polynomials satisfy

$$
\begin{array}{r}
\sum_{n=0}^{\infty} \frac{\left(1-a b c d q^{2 n-1}\right)\left(a b c d / q, t^{-1}\right)_{n}(d t)^{n}}{(1-a b c d / q)(q, a d, b d, c d, a b c d t ; q)_{n}} p_{n}(\cos \theta ; a, b, c, d \mid q) \\
=\frac{\left(a b c d, a d t, b d t, c d t, d e^{i \theta}, d e^{-i \theta} ; q\right)_{\infty}}{\left(a b c d t, a d, b d, c d, d t e^{i \theta}, d t e^{-i \theta} ; q\right)_{\infty}} .
\end{array}
$$


Professor Ismail kindly point out that this generating function follows from general theory of connection relations and from the connection relation in the Askey-Wilson memoir.

This generating function of the Askey-Wilson polynomials implies the following proposition [15, Corollary 4].

Proposition 1.7. The Askey-Wilson polynomials $p_{n}(\cos \theta ; a, b, c, d \mid q)$ are symmetric in $a, b, c$ and $d$.

Interchanging $a$ and $d$ in Proposition 1.6 and noting $p_{n}(\cos \theta ; a, b, c, d \mid q)$ are symmetric symmetric in $a$ and $d$, we deduce the following proposition.

Proposition 1.8. For $|a t|<1$, the Askey-Wilson polynomials satisfy

$$
\begin{array}{r}
\sum_{n=0}^{\infty} \frac{\left(1-a b c d q^{2 n-1}\right)\left(a b c d / q, t^{-1}\right)_{n}(a t)^{n}}{(1-a b c d / q)(q, a b, a c, a d, a b c d t ; q)_{n}} p_{n}(\cos \theta ; a, b, c, d \mid q) \\
=\frac{\left(a b c d, a b t, a c t, a d t, a e^{i \theta}, a e^{-i \theta} ; q\right)_{\infty}}{\left(a b c d t, a b, a c, a d, a t e^{i \theta}, a t e^{-i \theta} ; q\right)_{\infty}} .
\end{array}
$$

This generating function was used by us to give a new proof of the orthogonality for the Askey-Wilson polynomials [17, pp. 199-200].

In Section 2 of this paper we will use Proposition 1.6 and the orthogonality relation for the Askey-Wilson polynomials to give a new proof of the Nassrallah-Rahman integral. Section 3 is devoted to some inequalities for $q$-series. In Section 4, we will prove the following $q$-beta integral which has twelve parameters with base $q$.

Theorem 1.9. For $\alpha=a^{2} b c d r / q$ and $\max \{|a|,|b|,|c|,|d|,|r|,|s|,|t|,|h|,|z|\}<1$, we have

$$
\begin{gathered}
\int_{0}^{\pi} \frac{h(\cos 2 \theta ; 1 \mid q)}{h(\cos \theta ; a, b, c, d, r \mid q)}{ }_{4} \phi_{3}\left(\begin{array}{c}
a e^{i \theta}, a e^{-i \theta}, \beta, \delta \\
s, t, h
\end{array} ;, b c d r z\right) d \theta \\
=\frac{2 \pi(a b c d, a b c r, a b d r, a c d r ; q)_{\infty}}{(q, a b, a c, a d, a r, b c, b d, b r, c d, c r, d r, q \alpha ; q)_{\infty}} \\
\quad \times \sum_{n=0}^{\infty} \frac{\left(1-\alpha q^{2 n}\right)(\alpha, a b, a c, a d, a r ; q)_{n}}{(1-\alpha)(q, a b c d, a b c r, a b d r, a c d r ; q)_{n}}(-b c d r)^{n} q^{n(n-1) / 2} \\
\times{ }_{4} \phi_{3}\left(\begin{array}{c}
q^{-n}, \alpha q^{n}, \beta, \delta \\
s, t, h
\end{array} ;, q z\right) .
\end{gathered}
$$

The theorem also holds when $|z|=1$, provided the series on the right-hand side of the above equation converges absolutely for $|z|=1$.

Note that when $r=0$, the above integral immediately reduces to the Askey-Wilson integral in Proposition 1.2. In Section 5, we will show that Theorem 1.9] is equivalent to the following double $q$-series transformation formula.

Theorem 1.10. For $\alpha=a^{2} b c d r / q$ and $\max \{|a|,|b|,|c|,|d|,|r|,|s|,|t|,|h|,|z|\}<1$, we have

$$
\begin{gathered}
\sum_{n=0}^{\infty} \frac{(\beta, \delta, a b, a c, a d, a r ; q)_{n}(b c d r z)^{n}}{(q, s, t, h, a b c d, a b c r ; q)_{n}}{ }_{3} \phi_{2}\left(\begin{array}{c}
a b q^{n}, a c q^{n}, b c \\
a b c d q^{n}, a b c r q^{n}
\end{array} ; q, d r\right) \\
=\frac{(a b d r, a c r ; q)_{\infty}}{(d r, q \alpha ; q)_{\infty}} \sum_{n=0}^{\infty} \frac{\left(1-\alpha q^{2 n}\right)(\alpha, a b, a c, a d, a r ; q)_{n}}{(1-\alpha)(q, a b c d, a b c r, a b d r, a c d r ; q)_{n}}(-b c d r)^{n} q^{n(n-1) / 2} \\
\times{ }_{4} \phi_{3}\left(\begin{array}{c}
q^{-n}, \alpha q^{n}, \beta, \delta \\
s, t, h
\end{array} ;, q z\right) .
\end{gathered}
$$


The theorem also holds when $|z|=1$, provided the series on the right-hand side of the above equation converges absolutely for $|z|=1$.

Some interesting special cases of Theorem 1.9 will be discussed in Section 6 .

\section{A NeW Proof of the NassRallah-Rahman integral}

Nassrallah and Rahman [24, pp.192-194] used the integral representation of the sum of two non-terminating ${ }_{3} \phi_{2}$ series and the Askey-Wilson integral to find the following $q$-beta integral formula, which is a very general extension of Euler's integral representation of the classical ${ }_{2} F_{1}$ function. Ismail and Zhang [9] evaluate the Nassrallah-Rahman integral in a clever way by using the method of analytic continuation. In this section we will use Proposition 1.6 and the orthogonality relation for the Askey-Wilson polynomials to give a new proof of the Nassrallah-Rahman integral. Our proof is somewhat simpler than that of Nassrallah and Rahman.

Theorem 2.1. For $\{|a|,|b|,|c|,|u|,|v|\}<1$, we have the integral formula

$$
\begin{aligned}
& \int_{0}^{\pi} \frac{h(\cos 2 \theta ; 1 \mid q) h(\cos \theta ; d \mid q)}{h(\cos \theta ; a, b, c, u, v \mid q)} d \theta \\
& =\frac{2 \pi(a b c u, a b c v, a d, b d, c d ; q)_{\infty}}{(q, a b c d, a b, a c, a u, a v, b c, b u, b v, c u, c v ; q)_{\infty}} \\
& \quad \times{ }_{8} W_{7}(a b c d / q ; a b, a c, b c, d / u, d / v ; q, u v) .
\end{aligned}
$$

Proof. For the sake of brevity, we for the time being use $p_{n}(\cos \theta \mid q)$ to denote $p_{n}(\cos \theta ; a, b, c, d \mid q)$ and $\Delta_{n}(t)$ to denote

$$
\frac{\left(1-a b c d q^{2 n-1}\right)\left(a b c d / q, t^{-1}\right)_{n}(d t)^{n}}{(1-a b c d / q)(q, a d, b d, c d, a b c d t ; q)_{n}}
$$

Keeping this in mind, we can rewrite the equation in Proposition 1.6 in the form

$$
\sum_{n=0}^{\infty} \Delta_{n}(t) p_{n}(\cos \theta \mid q)=\frac{(a b c d, a d t, b d t, c d t ; q)_{\infty} h(\cos \theta ; d)}{(a b c d t, a d, b d, c d ; q)_{\infty} h(\cos \theta ; d t)}
$$

If $t$ is replaced by $s$ in the above equation and making the variable change $n \rightarrow m$, then, we obtain

$$
\sum_{m=0}^{\infty} \Delta_{m}(s) p_{m}(\cos \theta \mid q)=\frac{(a b c d, a d s, b d s, c d s ; q)_{\infty} h(\cos \theta ; d)}{(a b c d t, a d, b d, c d ; q)_{\infty} h(\cos \theta ; d s)} .
$$

Upon multiplying the two equations above together, we immediately conclude that

$$
\begin{aligned}
& \sum_{m=0}^{\infty} \sum_{n=0}^{\infty} \Delta_{m}(s) \Delta_{n}(t) p_{m}(\cos \theta \mid q) p_{n}(\cos \theta \mid q) \\
& =\frac{(a b c d, a b c d, a d s, a d t, b d s, b d t, c d s, c d t ; q)_{\infty} h(\cos \theta ; d, d \mid q)}{(a b c d s, a b c d t, a d, a d, b d, b d, c d, c d ; q)_{\infty} h(\cos \theta ; d s, d t)} .
\end{aligned}
$$

Multiplying the above equation by $W_{(a, b, c, d)}(\cos \theta \mid q)$ and then integrating with respect to $\theta$ over $[0, \pi]$, we deduce that

$$
\begin{aligned}
& \sum_{m=0}^{\infty} \sum_{n=0}^{\infty} \Delta_{m}(s) \Delta_{n}(t) \int_{0}^{\pi} p_{m}(\cos \theta \mid q) p_{n}(\cos \theta \mid q) W_{(a, b, c, d)}(\cos \theta \mid q) d \theta \\
& =\frac{(a b c d, a b c d, a d s, a d t, b d s, b d t, c d s, c d t ; q)_{\infty}}{(a b c d s, a b c d t, a d, a d, b d, b d, c d, c d ; q)_{\infty}} \int_{0}^{\pi} \frac{h(\cos 2 \theta ; 1) h(\cos \theta ; d \mid q)}{h(\cos \theta ; a, b, c, d s, d t \mid q)} d \theta
\end{aligned}
$$


Using the orthogonality relation for the Askey-Wilson polynomials to simplify the left-hand side of the above equation, we find that

$$
\begin{aligned}
& K(a, b, c, d \mid q) \sum_{n=0}^{\infty} \frac{(1-a b c d / q)(q, a b, a c, a d, b c, b d, c d ; q)_{n}}{\left(1-a b c d q^{2 n-1}\right)(a b c d / q ; q)_{n}} \Delta_{n}(s) \Delta_{n}(t) \\
& =K(a, b, c, d \mid q){ }_{8} W_{7}\left(a b c d / q ; a b, a c, b c, s^{-1}, t^{-1} ; q, s t d^{2}\right) .
\end{aligned}
$$

It follows that

$$
\begin{aligned}
& \int_{0}^{\pi} \frac{h(\cos 2 \theta ; 1) h(\cos \theta ; d \mid q)}{h(\cos \theta ; a, b, c, d s, d t \mid q)} d \theta \\
& =\frac{2 \pi(a b c d s, a b c d t, a d, b d, c d ; q)_{\infty}}{(q, a b c d, a b, a c, b c, a d s, b d s, c d s, a d t, b d t, c d t ; q)_{\infty}} \\
& \quad \times{ }_{8} W_{7}\left(a b c d / q ; a b, a c, b c, s^{-1}, t^{-1} ; q, s t d^{2}\right) .
\end{aligned}
$$

This completes the proof of Theorem 2.1 after replacing $d s$ by $u$ and $d t$ by $v$.

When $d=a b c u v$, using Proposition 1.4, the ${ }_{8} W_{7}(\cdot)$ series in the Nassrallah-Rahman integral reduces to

$$
{ }_{6} W_{5}(a b c d / q ; a b, a c, b c ; q, u v)=\frac{(a b c d, b c u v, a c u v, a b u v ; q)_{\infty}}{(a d, b d, c d, u v ; q)_{\infty}} .
$$

Hence Theorem 2.1 reduces to the following $q$-integral formula, which was first derived by Rahman [26, Eq.(2.1)].

Theorem 2.2. For $\{|a|,|b|,|c|,|u|,|v|\}<1$, we have the integral formula

$$
\begin{aligned}
& \int_{0}^{\pi} \frac{h(\cos 2 \theta ; 1 \mid q) h(\cos \theta ; a b c u v \mid q)}{h(\cos \theta ; a, b, c, u, v \mid q)} d \theta \\
& =\frac{2 \pi(a b c u, a b c v, a b u v, a c u v, b c u v ; q)_{\infty}}{(q, a b, a c, a u, a v, b c, b u, b v, c u, c v, u v ; q)_{\infty}} .
\end{aligned}
$$

Upon setting $d=0$ in the Nassrallah-Rahman integral in Theorem 2.1, we immediately arrive at the Ismail-Stanton-Viennot integral [7, Theorem 3.5].

Theorem 2.3. For $\{|a|,|b|,|c|,|u|,|v|\}<1$, we have the integral formula

$$
\begin{aligned}
& \int_{0}^{\pi} \frac{h(\cos 2 \theta ; 1 \mid q)}{h(\cos \theta ; a, b, c, u, v \mid q)} d \theta \\
& =\frac{2 \pi(a b c u, a b c v ; q)_{\infty}}{(q, a b, a c, a u, a v, b c, b u, b v, c u, c v ; q)_{\infty}}{ }_{3} \phi_{2}\left(\begin{array}{c}
a b, a c, b c \\
a b c u, a b c v
\end{array} ; q, u v\right) .
\end{aligned}
$$

\section{SOME INEQUALITIES FOR $q$-SERIES}

The following proposition is proved by us in [22, Proposition 3.1]. For completeness, we will repeat the proof of this proposition.

Proposition 3.1. If $0<q<1$ and $k$ is a nonnegative integer or $\infty$, a and $b$ are two nonnegative numbers such that $0 \leq b \leq 1$, then, we have

$$
(-a b ; q)_{k} \leq(-a ; q)_{\infty} .
$$

If we further assume that $0 \leq a \leq 1$, then, we have the inequality

$$
(a b ; q)_{k} \geq(a ; q)_{\infty} .
$$


Proof. Keeping that $0<q<1$ in mind, we find that for $j \in\{0,1, \ldots, k-1\}$,

$$
1+a b q^{j} \leq 1+a q^{j}
$$

Upon multiplying these $k$ inequalities together, we immediately deduce that

$$
(-a b ; q)_{k} \leq(-a ; q)_{k} .
$$

Since $\left(-a q^{k} ; q\right)_{\infty} \geq 1$, we multiply $\left(-a q^{k} ; q\right)_{\infty}$ to the right-hand side of the above inequality to arrive at the first inequality in the proposition. In the same way we can prove the second inequality. This completes the proof of Proposition 3.1 .

Proposition 3.2. If $\max \left\{\left|b_{1}\right|,\left|b_{2}\right|, \ldots,\left|b_{r}\right|\right\}<1,|x| \leq \lambda<1$ and $n$ is a nonnegative integer, then, we have

$$
q^{\left(\begin{array}{c}
n \\
2
\end{array}\right)}\left|{ }_{r+1} \phi_{r}\left(\begin{array}{c}
q^{-n}, a_{1} q^{n}, a_{2}, \ldots, a_{r} \\
b_{1}, \ldots b_{r}
\end{array} ;, q x\right)\right| \leq \frac{\left(-\left|a_{1} \lambda\right|,-q,-\left|a_{2}\right|, \ldots,-\left|a_{r}\right| ; q\right)_{\infty}}{\left(|\lambda|,\left|b_{1}\right|, \ldots,\left|b_{r}\right| ; q\right)_{\infty}} .
$$

Proof. By a simple evaluation, we easily find that (see, for example [5. Eq.(1.2.37)])

$$
\left(q^{-n} ; q\right)_{k}=(-1)^{k} q^{-n k+\left(\begin{array}{c}
k \\
2
\end{array}\right)}\left(1-q^{n}\right)\left(1-q^{n-1}\right) \cdots\left(1-q^{n-k+1}\right) .
$$

It follows that

$$
\begin{aligned}
& q^{\left(\begin{array}{c}
n \\
2
\end{array}\right)}{ }_{r+1} \phi_{r}\left(\begin{array}{c}
q^{-n}, a_{1} q^{n}, a_{2}, \ldots, a_{r} \\
b_{1}, \ldots b_{r}
\end{array} q, q x\right) \\
& =\sum_{k=0}^{n}(-1)^{k} q^{\left(\begin{array}{c}
n-k \\
2
\end{array}\right)} \frac{\left(q^{n-k+1}, a_{1} q^{n}, a_{2}, \ldots, a_{r} ; q\right)_{k}}{\left(q, b_{1}, \ldots, b_{r} ; q\right)_{k}} x^{k} .
\end{aligned}
$$

Keeping that $0<q<1$ in mind, noting that $0<q^{\left(\begin{array}{c}n-k \\ 2\end{array}\right)} \leq 1$ and using the triangle inequality, we immediately find that

$$
\begin{aligned}
& q^{\left(\begin{array}{c}
n \\
2
\end{array}\right)}\left|{ }_{r+1} \phi_{r}\left(\begin{array}{c}
q^{-n}, a_{1} q^{n}, a_{2}, \ldots, a_{r} \\
b_{1}, \ldots b_{r}
\end{array} q, q x\right)\right| \\
& \leq \sum_{k=0}^{n} \frac{\left|\left(q^{n-k+1}, a_{1} q^{n}, a_{2}, \ldots, a_{r} ; q\right)_{k}\right|}{(q ; q)_{k}\left|\left(q, b_{1}, \ldots, b_{r} ; q\right)_{k}\right|}|x|^{k} .
\end{aligned}
$$

Keeping that $0<q<1$ in mind, using the triangle inequality and the first inequality in Proposition 3.1 we deduce that

$$
\left|\left(a_{1} q^{n} ; q\right)_{k}\right| \leq\left(-\left|a_{1}\right| q^{n} ; q\right)_{k} \leq\left(-\left|a_{1}\right| ; q\right)_{k} .
$$

Noting that $0<q^{n-k} \leq 1$ for $k \in\{0,1, \ldots, n\}$, using the triangle inequality and the first inequality in Proposition 3.1 we find that

$$
\left(q^{n-k+1} ; q\right)_{k} \leq\left(-q^{n-k+1} ; q\right)_{k} \leq(-q ; q)_{\infty} .
$$

Upon making the triangle inequality and noting that $\left(1+\left|a_{j}\right| q^{l}\right) \geq 1$, we see that, for $j \in\{2,3, \ldots, r\}$,

$$
\left|\left(a_{j} ; q\right)_{k}\right| \leq \prod_{l=0}^{k-1}\left(1+\left|a_{j}\right| q^{l}\right) \leq \prod_{l=0}^{\infty}\left(1+\left|a_{j}\right| q^{l}\right) .
$$

Using the second inequality in Proposition 3.1, we find that, for $j \in\{1,2, \ldots, r\}$,

$$
\left|\left(b_{j} ; q\right)_{k}\right| \geq\left(\left|b_{j}\right| ; q\right)_{\infty} .
$$


It follows that

$$
\frac{\left|\left(q^{n-k+1}, a_{1} q^{n}, a_{2}, \ldots, a_{r} ; q\right)_{k}\right|}{(q ; q)_{k}\left|\left(q, b_{1}, \ldots, b_{r} ; q\right)_{k}\right|}|x|^{k} \leq \frac{\left(-q,-\left|a_{2}\right|, \ldots,-\left|a_{r}\right| ; q\right)_{\infty}\left(-\left|a_{1}\right| ; q\right)_{k}}{\left(\left|b_{1}\right|, \ldots,\left|b_{r}\right| ; q\right)_{\infty}(q ; q)_{k}}|\lambda|^{k} .
$$

Using this inequality the triangle inequality, we conclude that

$$
\begin{aligned}
& q^{\left(\begin{array}{c}
n \\
2
\end{array}\right)}\left|{ }_{r+1} \phi_{r}\left(\begin{array}{c}
q^{-n}, a_{1} q^{n}, a_{2}, \ldots, a_{r} \\
b_{1}, \ldots b_{r}
\end{array} ; q, q x\right)\right| \\
& \leq \frac{\left(-q,-\left|a_{2}\right|, \ldots,-\left|a_{r}\right| ; q\right)_{\infty}}{\left(\left|b_{1}\right|, \ldots,\left|b_{r}\right| ; q\right)_{\infty}} \sum_{k=0}^{\infty} \frac{\left(-\left|a_{1}\right| ; q\right)_{k}|\lambda|^{k}}{(q ; q)_{k}} .
\end{aligned}
$$

Upon applying the $q$-binomial theorem to the right-hand side of the above inequality, we complete the proof of Proposition 3.2 .

Proposition 3.3. If $k$ is a nonnegative integer, then, for $\max \{|a|,|b|,|c|,|d|,|u|\}<1, A_{k}(\theta)$ is bounded on $[-\pi, \pi]$, where $A_{k}(\theta)$ is given by

$$
A_{k}(\theta)=\frac{h(\cos 2 \theta ; 1 \mid q) h(\cos \theta ; w \mid q)\left(t e^{i \theta}, t e^{-i \theta} ; q\right)_{k}}{h(\cos \theta ; a, b, c, d, u \mid q)} .
$$

\section{THE PROOF OF THEOREM 1.9}

The following general $q$-transformation formula is proved by us in [17, Theorem 1.6].

Proposition 4.1. For an arbitrary sequence $\left\{A_{n}\right\}$ of complex numbers, under suitable convergence conditions, we have

$$
\begin{aligned}
& \frac{(\alpha q, \alpha u v / q ; q)_{\infty}}{(\alpha u, \alpha v ; q)_{\infty}} \sum_{n=0}^{\infty} A_{n}(q / u ; q)_{n}(\alpha u)^{n} \\
& =\sum_{n=0}^{\infty} \frac{\left(1-\alpha q^{2 n}\right)(\alpha, q / u, q / v ; q)_{n}(-\alpha u v / q)^{n} q^{n(n-1) / 2}}{(1-\alpha)(q, \alpha u, \alpha v ; q)_{n}} \\
& \times \sum_{k=0}^{n} \frac{\left(q^{-n}, \alpha q^{n} ; q\right)_{k}\left(q^{2} / v\right)^{k}}{(q / v ; q)_{k}} A_{k} .
\end{aligned}
$$

It is easily seen that by choosing

$$
A_{k}=\frac{(q / v, \beta, \delta ; q)_{k}(v z / q)^{k}}{(q, s, t, h ; q)_{k}}
$$

in Proposition 4.1, we can derive the following proposition, which was used to prove may of the Hecke-type series identities in [18].

Proposition 4.2. For $\max \{|\alpha a|,|\alpha b|,|\alpha u v z / q|\}<1$, we have the q-formula

$$
\begin{aligned}
& \frac{(\alpha q, \alpha u v / q ; q)_{\infty}}{(\alpha u, \alpha v ; q)_{\infty}}{ }_{4} \phi_{3}\left(\begin{array}{c}
q / u, q / v, \beta, \delta \\
s, t, h
\end{array} ; q, \frac{\alpha u v z}{q}\right) \\
& =\sum_{n=0}^{\infty} \frac{\left(1-\alpha q^{2 n}\right)(\alpha, q / u, q / v ; q)_{n}(-\alpha u v / q)^{n} q^{n(n-1) / 2}}{(1-\alpha)(q, \alpha u, \alpha v ; q)_{n}} \\
& \times{ }_{4} \phi_{3}\left(\begin{array}{c}
q^{-n}, a^{2} b c d r q^{n-1}, \beta, \delta \\
s, t, h
\end{array}{ }^{n}, q z\right),
\end{aligned}
$$

provided the right-hand side of the above equation is an absolutely convergent series. 
Now we begin prove Theorem 1.9 by using Propositions 3.1 , 3.2, 3.3 and 4.2, as well as the Nassrallah- Rahman integral in Theorem 2.2.

Proof. For notational simplicity, we will for the time being make use of $B_{n}$ to denote

$$
\begin{gathered}
\frac{\left(1-\alpha q^{2 n}\right)(\alpha, q / u, q / v ; q)_{n}(-\alpha u v / q)^{n} q^{n(n-1) / 2}}{(1-\alpha)(q, \alpha u, \alpha v ; q)_{n}} \\
\times{ }_{4} \phi_{3}\left(\begin{array}{c}
q^{-n}, a^{2} b c d r q^{n-1}, \beta, \delta \\
s, t, h
\end{array} ; q, q z\right) .
\end{gathered}
$$

Upon taking $\alpha=a^{2} b c d r / q, u=q e^{i \theta} / a$ and $v=q e^{-i \theta} / a$ in Proposition 4.2, we find that

$$
\begin{aligned}
& \sum_{n=0}^{\infty} \frac{\left(a e^{i \theta}, a e^{-i \theta} ; q\right)_{n}}{\left(a b c d r e^{i \theta}, a b c d r e^{-i \theta} ; q\right)_{n}} B_{n} \\
& =\frac{\left(a^{2} b c d r, b c d r ; q\right)_{\infty}}{\left(a b c d r e^{i \theta}, a b c d r e^{-i \theta} ; q\right)_{\infty}}{ }_{4} \phi_{3}\left(\begin{array}{c}
a e^{i \theta}, a e^{-i \theta}, \beta, \delta \\
s, t, h
\end{array}{ }^{i q, b c d r z}\right) .
\end{aligned}
$$

Using the triangular inequality and a simple calculation, we deduce that

$$
\left|\left(a e^{i \theta}, a e^{-i \theta} ; q\right)_{n}\right| \leq(-|a| ; q)_{\infty}^{2} .
$$

Keeping that $\max \{|a|,|b|,|c|,|d|,|r|\}<1$ in mind and using the triangular inequality, we have

$$
\left|\left(a b c d r e^{i \theta}, a b c d r e^{-i \theta} ; q\right)_{n}\right| \geq(|a b c d r| ; q)_{\infty}^{2}
$$

It follows that

$$
\left|\frac{\left(a e^{i \theta}, a e^{-i \theta} ; q\right)_{n}}{\left(a b c d r e^{i \theta}, a b c d r e^{-i \theta} ; q\right)_{n}}\right| \leq \frac{(-|a| ; q)_{\infty}^{2}}{(|a b c d r| ; q)_{\infty}^{2}} .
$$

Assuming that $0<\lambda<1$ and $|z| \leq \lambda$, then by Proposition 3.2 , we find that

$$
q^{n(n-1) / 2}\left|{ }_{4} \phi_{3}\left(\begin{array}{c}
q^{-n}, a^{2} b c d r q^{n-1}, \beta, \delta \\
s, t, h
\end{array} ; q, q z\right)\right|
$$

is bounded by some constant independent of $n$ and $z$. Hence by the Hence by the ratio test we know that the series on the left-hand side of (4.1) is an absolutely and uniformly convergent series of two variables $z$ and $\theta$ for $\max \{|a|,|b|,|c|,|d|,|r|\}<1$ and $|z| \leq \lambda$, where $0<\lambda<1$.

Using Proposition 3.3, with $t=0$, we know that the following function of $\theta$ is bounded by some constant independent of $\theta$ :

$$
\frac{h(\cos 2 \theta ; 1 \mid q) h(\cos \theta ; a b c d r \mid q)}{h(\cos \theta ; a, b, c, d, r \mid q)} .
$$

Upon multiplying both sides of (4.1) by the above function of $\theta$, using the identity $(x ; q)_{n}\left(x q^{n} ; q\right)_{\infty}=$ $(x ; q)_{\infty}$ in [5, Eq.(1.2.30)], we obtain

$$
\begin{aligned}
& \frac{h(\cos 2 \theta ; 1 \mid q)}{h(\cos \theta ; a, b, c, d, r \mid q)}{ }_{4} \phi_{3}\left(\begin{array}{c}
a e^{i \theta}, a e^{-i \theta}, \beta, \delta \\
s, t, h
\end{array} ; q, b c d r z\right) \\
& =\frac{1}{\left(a^{2} b c d r, b c d r ; q\right)_{\infty}} \sum_{n=0}^{\infty} \frac{h(\cos 2 \theta ; 1 \mid q) h\left(\cos \theta ; a b c d r q^{n} \mid q\right)}{h(\cos \theta ; a, b, c, d, r \mid q)} B_{n} .
\end{aligned}
$$


Through the above discussion, we know that the right-hand side of the above equation is convergent uniformly and absolutely on $[0, \pi]$. Hence we can integrate the above equation term by term over $[0, \pi]$ to obtain

$$
\begin{aligned}
& \int_{0}^{\pi} \frac{h(\cos 2 \theta ; 1 \mid q)}{h(\cos \theta ; a, b, c, d, r \mid q)}{ }_{4} \phi_{3}\left(\begin{array}{c}
a e^{i \theta}, a e^{-i \theta}, \beta, \delta \\
s, t, h
\end{array} ;, b c d r z\right) d \theta \\
& =\frac{1}{\left(a^{2} b c d r, b c d r ; q\right)_{\infty}} \sum_{n=0}^{\infty} B_{n} \int_{0}^{\pi} \frac{h(\cos 2 \theta ; 1 \mid q) h\left(\cos \theta ; a b c d r q^{n} \mid q\right)}{h(\cos \theta ; a, b, c, d, r \mid q)} d \theta
\end{aligned}
$$

Using the Nassrallah-Rahman $q$-beta integral in Theorem 2.2, we immediately conclude that

$$
\begin{aligned}
& \int_{0}^{\pi} \frac{h(\cos 2 \theta ; 1 \mid q) h\left(\cos \theta ; a b c d r q^{n} \mid q\right)}{h(\cos \theta ; a, b, c, d, r \mid q)} d \theta \\
& =\frac{2 \pi(a b c d, a b c r, a b d r, a c d r, b c d r ; q)_{\infty}(a b, a c, a d, a r ; q)_{n}}{(q, a b, a c, a d, a r, b c, b d, b r, c d, c r, d r ; q)_{\infty}(a b c d, a b c r, a b d r, a c d r ; q)_{n}} .
\end{aligned}
$$

Combining the two equations above we complete the proof of Theorem 1.9. From the process of proof of Theorem 1.9, we find that this theorem also holds when $|z|=1$, provided the series on the right-hand side of the above equation converges absolutely for $|z|=1$.

\section{THE PRoOF OF TheOREM 1.10}

Proof. It follows that from (4.2), by the ratio test, we find that the following series is absolutely and uniformly convergent on $[0, \pi]$ when $|b c d r z|<1$ :

$$
{ }_{4} \phi_{3}\left(\begin{array}{c}
a e^{i \theta}, a e^{-i \theta}, \beta, \delta \\
s, t, h
\end{array} ; q, b c d r z\right) .
$$

Using Proposition 3.3, with $t=w=0$, we know that the following function of $\theta$ is bounded by some constant independent of $\theta$ :

$$
\frac{h(\cos 2 \theta ; 1 \mid q)}{h(\cos \theta ; a, b, c, d, r \mid q)} .
$$

Hence we can evaluate the integral on the left-hand side of the equation in Theorem 1.9 term by term to obtain

$$
\begin{aligned}
& \int_{0}^{\pi} \frac{h(\cos 2 \theta ; 1 \mid q)}{h(\cos \theta ; a, b, c, d, r \mid q)}{ }^{4} \phi_{3}\left(\begin{array}{c}
a e^{i \theta}, a e^{-i \theta}, \beta, \delta \\
s, t, h
\end{array} ;, b c d r z\right) d \theta \\
& =\sum_{n=0}^{\infty} \frac{(\beta, \delta ; q)_{n}(b c d r z)^{n}}{(q, s, t, h ; q)_{n}} \int_{0}^{\pi} \frac{h(\cos 2 \theta ; 1 \mid q)}{h\left(\cos \theta ; a q^{n}, b, c, d, r \mid q\right)} d \theta .
\end{aligned}
$$

Using the Ismail-Stanton-Viennot integral in Theorem 2.3, we immediately deduce that

$$
\begin{aligned}
& \int_{0}^{\pi} \frac{h(\cos 2 \theta ; 1 \mid q)}{h\left(\cos \theta ; a q^{n}, b, c, d, r \mid q\right)} d \theta \\
& =\frac{2 \pi\left(a b c d q^{n}, a b c r q^{n} ; q\right)_{\infty}}{\left(q, a b q^{n}, a c q^{n}, a d q^{n}, a r q^{n}, b c, b d, b r, c d, c r ; q\right)_{\infty}}{ }_{3} \phi_{2}\left(\begin{array}{c}
a b q^{n}, a c q^{n}, b c \\
a b c d q^{n}, a b c r q^{n}
\end{array} ;, d r\right) .
\end{aligned}
$$


It follows that

$$
\begin{aligned}
& \int_{0}^{\pi} \frac{h(\cos 2 \theta ; 1 \mid q)}{h(\cos \theta ; a, b, c, d, r \mid q)}{ }_{4} \phi_{3}\left(\begin{array}{c}
a e^{i \theta}, a e^{-i \theta}, \beta, \delta \\
s, t, h
\end{array} ; q, b c d r z\right) d \theta \\
& =\frac{2 \pi(a b c d, a b c r ; q)_{\infty}}{(q, a b, a c, a d, a r, b c, b d, b r, c d, c r ; q)_{\infty}} \\
& \quad \times \sum_{n=0}^{\infty} \frac{(a b, a c, a d, a r, \beta, \delta ; q)_{n}(b c d r z)^{n}}{(q, a b c d, a b c r, s, t, h ; q)_{n}}{ }_{3} \phi_{2}\left(\begin{array}{c}
a b q^{n}, a c q^{n}, b c \\
a b c d q^{n}, a b c r q^{n}
\end{array} ; q, d r\right) .
\end{aligned}
$$

Equating this with the right-hand side of the equation in Theorem 1.9, we complete the proof of Theorem 1.10 .

\section{SOME SPECIAL CASES OF TheOREM 1.9}

Theorem 6.1. For $\max \{|a|,|b|,|c|,|d|,|r|\}<1$, we have

$$
\begin{aligned}
& \int_{0}^{\pi} \frac{h(\cos 2 \theta ; 1 \mid q) h(\cos \theta ; a b c d r z \mid q)}{h(\cos \theta ; a, b, c, d, r \mid q)} d \theta \\
& =\frac{2 \pi\left(a b c d, a b c r, a b d r, a c d r, a^{2} b c d r z, b c d r z ; q\right)_{\infty}}{\left(q, a b, a c, a d, a r, b c, b d, b r, c d, c r, d r, a^{2} b c d r ; q\right)_{\infty}} \\
& \quad \times{ }_{8} W_{7}\left(a^{2} b c d r / q ; a b, a c, a d, a r, 1 / z ; b c d r z\right) .
\end{aligned}
$$

This integral is slightly different in form from the Nassrallah-Rahman $q$-beta integral in Theorem 2.1. Putting $z=1$, noting that $(1 ; q)_{k}=\delta_{0, k}$, the above equation immediately reduces to the Nassrallah-Rahman $q$-beta integral in Theorem 2.2.

Now we prove Theorem 6.1 by using Theorem 1.9, the $q$-Gauss summation formula and $q$-Chu-Vandermonde summation formula.

Proof. When $s=a^{2} b c d r z$ and $(\beta, \delta)=(t, h)$, the ${ }_{4} \phi_{3}$ series under the integral sign in Theorem 1.9 reduces to

$$
{ }_{2} \phi_{1}\left(\begin{array}{c}
a e^{i \theta}, a e^{-i \theta} \\
a^{2} b c d r z
\end{array} ; q, b c d r z\right)=\frac{\left(a b c d r z e^{i \theta}, a b c d r z e^{-i \theta} ; q\right)_{\infty}}{\left(a^{2} b c d r z, b c d r z ; q\right)_{\infty}}
$$

by the $q$-Gauss summation [5, Eq.(1.5.1)], and the ${ }_{4} \phi_{3}$ series on the right-hand side of the equation in Theorem 1.9 becomes

$$
{ }_{2} \phi_{1}\left(\begin{array}{c}
q^{-n}, a^{2} b c d r q^{n-1} \\
a^{2} b c d r z
\end{array} ; q, q z\right)=\frac{(1 / z ; q)_{n}(-z)^{n}}{\left(a^{2} b c d r z ; q\right)_{n}} q^{-n(n-1) / 2}
$$

by the $q$-Chu-Vandermonde summation [5, Eq.(1.5.2)]. This completes the proof of Theorem 6.1.

The corresponding series form of Theorem 6.1 is stated in the following theorem, which is equivalent to the formula due to Al-Salam and Ismail [1, Eq.(4.4)].

Theorem 6.2. For $\max \{|a|,|b|,|c|,|d|,|r|\}<1$, we have

$$
\begin{aligned}
& \sum_{n=0}^{\infty} \frac{(a b, a c, a d, a r ; q)_{n}(b c d r z)^{n}}{\left(q, a^{2} b c d r z, a b c d, a b c r ; q\right)_{n}}{ }_{3} \phi_{2}\left(\begin{array}{c}
a b q^{n}, a c q^{n}, b c \\
a b c d q^{n}, a b c r q^{n}
\end{array} ;, d r\right) \\
& =\frac{(a b d r, a c d r ; q)_{\infty}}{\left(d r, a^{2} b c d r ; q\right)_{\infty}}{ } W_{7}\left(a^{2} b c d r / q ; a b, a c, a d, a r, 1 / z ; b c d r z\right) .
\end{aligned}
$$


Theorem 6.3. For $\alpha=a^{2} b c d r / q$ and $\max \{|a|,|b|,|c|,|d|,|r|\}<1$, we have

$$
\begin{aligned}
& \int_{0}^{\pi} \frac{h(\cos 2 \theta ; 1 \mid q)}{h(\cos \theta ; a, b, c, d, r \mid q)}{ }_{3} \phi_{2}\left(\begin{array}{c}
a e^{i \theta}, a e^{-i \theta}, \alpha u v / q \\
\alpha u, \alpha v
\end{array} ;, b c d r\right) d \theta \\
& =\frac{2 \pi(a b c d, a b c r, a b d r, a c d r ; q)_{\infty}}{\left(q, a b, a c, a d, a r, b c, b d, b r, c d, c r, d r, a^{2} b c d r ; q\right)_{\infty}} \\
& \quad \times \sum_{n=0}^{\infty} \frac{\left(1-\alpha q^{2 n}\right)(\alpha, a b, a c, a d, a r, q / u, q / v ; q)_{n}}{(1-\alpha)(q, a b c d, a b c r, a b d r, a c d r, \alpha u, \alpha v ; q)_{n}}\left(-\frac{\alpha b c d r u v}{q}\right)^{n} q^{n(n-1) / 2} .
\end{aligned}
$$

Proof. Noting that $\alpha=a^{2} b c d r / q$, choosing $s=\alpha u, t=\alpha v, \beta=\alpha u v / q$ and $\delta=h, z=1$ in Theorem 1.9, we deduce that

$$
\begin{gathered}
\int_{0}^{\pi} \frac{h(\cos 2 \theta ; 1 \mid q)}{h(\cos \theta ; a, b, c, d, r \mid q)}{ }_{3} \phi_{2}\left(\begin{array}{c}
a e^{i \theta}, a e^{-i \theta}, \alpha u v / q \\
\alpha u, \alpha v
\end{array} q, b c d r\right) d \theta \\
=\frac{2 \pi(a b c d, a b c r, a b d r, a c d r ; q)_{\infty}}{\left(q, a b, a c, a d, a r, b c, b d, b r, c d, c r, d r, a^{2} b c d r ; q\right)_{\infty}} \\
\times \sum_{n=0}^{\infty} \frac{\left(1-\alpha q^{2 n}\right)(\alpha, a b, a c, a d, a r ; q)_{n}}{(1-\alpha)(q, a b c d, a b c r, a b d r, a c d r ; q)_{n}}(-b c d r)^{n} q^{n(n-1) / 2} \\
\times{ }_{3} \phi_{2}\left(\begin{array}{c}
q^{-n}, \alpha q^{n}, \alpha u v / q \\
\alpha u, \alpha v
\end{array} q\right) .
\end{gathered}
$$

By the well-known $q$-Pfaff-Saalschütz formula [5, Eq.(1.7.2)], we find that

$$
{ }_{3} \phi_{2}\left(\begin{array}{c}
q^{-n}, \alpha q^{n}, \alpha u v / q \\
\alpha u, \alpha v
\end{array} ; q, q\right)=\frac{(q / u, q / v ; q)_{n}}{(\alpha u, \alpha v ; q)_{n}}\left(\frac{\alpha u v}{q}\right)^{n} .
$$

Upon combining the two equations above we complete the proof of Theorem 6.3

The corresponding series form of Theorem 6.3 is stated in the following theorem.

Theorem 6.4. For $\alpha=a^{2} b c d r / q$ and $\max \{|a|,|b|,|c|,|d|,|r|\}<1$, we have

$$
\begin{aligned}
& \sum_{n=0}^{\infty} \frac{(\alpha u v / q, a b, a c, a d, a r ; q)_{n}(b c d r)^{n}}{(q, \alpha u, \alpha v, a b c d, a b c r ; q)_{n}} 3 \phi_{2}\left(\begin{array}{c}
a b q^{n}, a c q^{n}, b c \\
a b c d q^{n}, a b c r q^{n}
\end{array} ;, d r\right) \\
& =\frac{(a b d r, a c d r ; q)_{\infty}}{(d r, q \alpha ; q)_{\infty}} \\
& \quad \times \sum_{n=0}^{\infty} \frac{\left(1-\alpha q^{2 n}\right)(\alpha, a b, a c, a d, a r, q / u, q / v ; q)_{n}}{(1-\alpha)(q, a b c d, a b c r, a b d r, a c d r, \alpha u, \alpha v ; q)_{n}}\left(-\frac{\alpha b c d r u v}{q}\right)^{n} q^{n(n-1) / 2} .
\end{aligned}
$$

Theorem 6.5. For $\alpha=a^{2} b c d r / q$ and $\max \{|a|,|b|,|c|,|d|,|r|\}<1$, we have

$$
\begin{aligned}
& \int_{0}^{\pi} \frac{h(\cos 2 \theta ; 1 \mid q)}{h(\cos \theta ; a, b, c, d, r \mid q)}{ }^{3} \phi_{2}\left(\begin{array}{l}
a e^{i \theta}, a e^{-i \theta}, 0 \\
\sqrt{q \alpha},-\sqrt{q \alpha}
\end{array} q, b c d r\right) d \theta \\
& =\frac{2 \pi(a b c d, a b c r, a b d r, a c d r ; q)_{\infty}}{\left(q, a b, a c, a d, a r, b c, b d, b r, c d, c r, d r, a^{2} b c d r ; q\right)_{\infty}} \\
& \quad \times \sum_{n=0}^{\infty} \frac{\left(1-\alpha q^{4 n}\right)(\alpha, a b, a c, a d, a r ; q)_{2 n}\left(q ; q^{2}\right)_{n}}{(1-\alpha)(q, a b c d, a b c r, a b d r, a c d r ; q)_{2 n}\left(q \alpha ; q^{2}\right)_{n}}(-\alpha)^{n}(b c d r)^{2 n} q^{3 n^{2}-n}
\end{aligned}
$$


Proof. It is easily seen that the identity of Verma and Jain [28, Eq.(2.28)] is equivalent to the summation

$$
{ }_{3} \phi_{2}\left(\begin{array}{ll}
q^{-n}, \alpha q^{n}, 0 \\
\sqrt{q \alpha},-\sqrt{q \alpha}
\end{array} ;, q\right)= \begin{cases}0 & \text { if } n \text { is odd } \\
(-1)^{l} q^{l^{2}} \frac{\left(q ; q^{2}\right)_{l} \alpha^{l}}{\left(q \alpha ; q^{2}\right)_{l}}, & \text { if } n=2 l\end{cases}
$$

Using this summation to Theorem [1.9, we complete the proof of Theorem 6.5,

The corresponding series form of Theorem 6.5 is stated in the following theorem.

Theorem 6.6. For $\alpha=a^{2} b c d r / q$ and $\max \{|a|,|b|,|c|,|d|,|r|\}<1$, we have

$$
\begin{aligned}
& \sum_{n=0}^{\infty} \frac{(a b, a c, a d, a r ; q)_{n}(b c d r)^{n}}{(q, \sqrt{q \alpha},-\sqrt{q \alpha}, a b c d, a b c r ; q)_{n}} 3 \phi_{2}\left(\begin{array}{c}
a b q^{n}, a c q^{n}, b c \\
a b c d q^{n}, a b c r q^{n}
\end{array} ;, d r\right) \\
& =\frac{(a b d r, a c d r ; q)_{\infty}}{(d r, q \alpha ; q)_{\infty}} \\
& \quad \times \sum_{n=0}^{\infty} \frac{(-\alpha)^{n}\left(1-\alpha q^{4 n}\right)(\alpha, a b, a c, a d, a r ; q)_{2 n}\left(q ; q^{2}\right)_{n}}{(1-\alpha)(q, a b c d, a b c r, a b d r, a c d r ; q)_{2 n}\left(q \alpha ; q^{2}\right)_{n}}(b c d r)^{2 n} q^{3 n^{2}-n}
\end{aligned}
$$

Theorem 6.7. For $\alpha=a^{2} b c d r / q$ and $\max \{|a|,|b|,|c|,|d|,|r|\}<1$, we have

$$
\begin{aligned}
& \int_{0}^{\pi} \frac{h(\cos 2 \theta ; 1 \mid q)}{h(\cos \theta ; a, b, c, d, r \mid q)}{ }_{4} \phi_{3}\left(\begin{array}{c}
a e^{i \theta}, a e^{-i \theta}, \sqrt{\lambda},-\sqrt{\lambda} \\
\sqrt{q \alpha},-\sqrt{q \alpha}, \lambda
\end{array} ;, b c d r\right) d \theta \\
& =\frac{2 \pi(a b c d, a b c r, a b d r, a c d r ; q)_{\infty}}{\left(q, a b, a c, a d, a r, b c, b d, b r, c d, c r, d r, a^{2} b c d r ; q\right)_{\infty}} \\
& \quad \times \sum_{n=0}^{\infty} \frac{\left(1-\alpha q^{4 n}\right)(\alpha, a b, a c, a d, a r ; q)_{2 n}\left(q, q \alpha / \lambda ; q^{2}\right)_{n}}{(1-\alpha)(q, a b c d, a b c r, a b d r, a c d r ; q)_{2 n}\left(q \alpha, q \lambda ; q^{2}\right)_{n}}(b c d r)^{2 n} \lambda^{n} q^{2 n^{2}-2 n} .
\end{aligned}
$$

Proof. Recall that the $q$-Watson formula due to Andrews [2] states that

$$
{ }_{4} \phi_{3}\left(\begin{array}{c}
q^{-n}, \alpha q^{n}, \sqrt{\lambda},-\sqrt{\lambda} \\
\sqrt{q \alpha},-\sqrt{q \alpha}, \lambda
\end{array} ; q, q\right)= \begin{cases}0 & \text { if } n \text { is odd } \\
\frac{\left(q, \alpha q / \lambda ; q^{2}\right)_{n / 2} \lambda^{n / 2}}{\left(q \alpha, q \lambda ; q^{2}\right)_{n / 2}}, & \text { if } n \text { is even. }\end{cases}
$$

For an alternative proof of this identity, please see [19, Proposition 8.3]. Using this equation to Theorem 1.9, we complete the proof of Theorem 6.7.

The corresponding series form of Theorem 6.7 is stated in the following theorem.

Theorem 6.8. For $\alpha=a^{2} b c d r / q$ and $\max \{|a|,|b|,|c|,|d|,|r|\}<1$, we have

$$
\begin{aligned}
& \sum_{n=0}^{\infty} \frac{(\sqrt{\lambda},-\sqrt{\lambda}, a b, a c, a d, a r ; q)_{n}(b c d r)^{n}}{(q, \sqrt{q \alpha},-\sqrt{q \alpha}, \lambda, a b c d, a b c r ; q)_{n}}{ }_{3} \phi_{2}\left(\begin{array}{c}
a b q^{n}, a c q^{n}, b c \\
a b c d q^{n}, a b c r q^{n}
\end{array} ; q, d r\right) \\
& =\frac{(a b d r, a c d r ; q)_{\infty}}{(d r, q \alpha ; q)_{\infty}} \\
& \quad \times \sum_{n=0}^{\infty} \frac{\left(1-\alpha q^{4 n}\right)(\alpha, a b, a c, a d, a r ; q)_{2 n}\left(q, q \alpha / \lambda ; q^{2}\right)_{n}}{(1-\alpha)(q, a b c d, a b c r, a b d r, a c d r ; q)_{2 n}\left(q \alpha, q \lambda ; q^{2}\right)_{n}}(b c d r)^{2 n} \lambda^{n} q^{2 n^{2}-2 n} .
\end{aligned}
$$




\section{ACKNowledgments}

I am grateful to the anonymous referee and the editor for careful reading of the manuscript and many invaluable suggestions and comments.

\section{REFERENCES}

[1] W. A. Al-Salam, M. E. H. Ismail, $q$-beta integrals and the $q$-Hermite polynomials. Pacific J. Math. 135 (1988) 209-221.

[2] G. E. Andrews, On $q$-analogues of the Watson and Whipple summations, SIAM J. Math. Anal. 7 (1976) $332-336$.

[3] R. Askey, An elementary evaluation of a beta type integral, Indian J. Pure. Appl. Math., 14 (1983) $892-895$.

[4] R. Askey and J. Wilson, Some basic hypergeometric orthogonal polynomials that generalize Jacobi polynomials, Memoirs. Amer. Math. Soc. 54, No. 319 (1985).

[5] G. Gasper and M. Rahman, Basic Hypergeometric series, 2nd ed., Cambridge Univ. Press, Cambridge, 2004.

[6] M. E. H. Ismail, J. A. Wilson, Asymptotic and generating relations for the $q$-Jacobi and ${ }_{4} \phi_{3}$ polynomials, J. Approx. Theory 36 (1) (1982) 43-54.

[7] M. E. H. Ismail, D. Stanton and G. Viennot, The combinatorics of $q$-Hermite polynomials and the Askey-Wilson integral, Europ. J. Combin. 8 (1987) 379-392.

[8] M. E. H. Ismail, M. Rahman, and S. K. Suslov, Some summation theorems and transformations for $q$-series, Can. J. Math. 49 (1997) 543-567.

[9] M.E.H. Ismail, R. Zhang, New proofs of some $q$-series results, in: Theory and Applications of Special Functions, in: Dev. Math., vol. 13, Springer, New York, 2005, pp. 285-299.

[10] M.E.H. Ismail, Classical and Quantum Orthogonal Polynomials in One Variable, Encyclopedia of Mathematics and its Applications, vol. 98, Cambridge University Press, Cambridge, 2009.

[11] E.G. Kalnins, W. Miller, Symmetry techniques for $q$-series: Askey-Wilson polynomials. Rocky Mt. J. Math. 19 (1989) 233-240.

[12] R. Koekoek, P.A. Lesky, R.F. Swarttouw, Hypergeometric Orthogonal Polynomials and Their qAnalogues, Springer-Verlag, Berlin, 2010.

[13] Z.-G. Liu, $q$-Hermite polynomials and a $q$-beta integral. Northeast. Math. J. 13 (1997) 361-366.

[14] Z.-G. Liu, An identity of Andrews and the Askey-Wilson integral. Ramanujan J. 19 (2009) 115-119.

[15] Z.-G. Liu, An extension of the non-terminating ${ }_{6} \phi_{5}$ summation and the Askey-Wilson polynomials, J. Difference Equ. Appl. 17 (2011) 1401-1411.

[16] Z.-G. Liu, On the $q$-partial differential equations and $q$-series, The Legacy of Srinivasa Ramanujan, Ramanujan Math. Soc. Lect. Notes Ser., vol. 20, pp. 213-250. Ramanujan Math. Soc., Mysore, 2013.

[17] Z.-G, Liu, A q-series expansion formula and the Askey-Wilson polynomials, Ramanujan J. 30 (2013) $193-210$.

[18] Z.-G. Liu, On the q-derivative and q-series expansion, Int. J. Number Theory 9 (2013) 2069-2089.

[19] Z.-G. Liu, A $q$-summation formula, the continuous $q$-Hahn polynomials and the big $q$-Jacobi polynomials. J. Math. Anal. Appl. 419 (2014) 1045-1064.

[20] Z.-G. Liu and J. Zeng, Two expansion formulas involving the Rogers-Szegő polynomials with applications, Int. J. Number Theory 11 (2015) 507-525.

[21] Z.-G, Liu, On a reduction formula for a kind of double $q$-integrals. Symmetry 8 (2016) Art. 44, 16 pp.

[22] Z.-G. Liu, Extensions of Ramanujan's reciprocity theorem and the Andrews-Askey integral, J. Math. Anal. Appl. 443 (2016) 1110-1129.

[23] Z.-G. Liu, On a reduction formula for a kind of double $q$-integrals. Symmetry 8 (2016), no. 6, Art. 44, $16 \mathrm{pp}$.

[24] B. Nassrallah, M. Rahman, Projection Formulas, a Reproducing Kernel and a Generating Function for $q$-Wilson Polynomials, SIAM J. Math. Anal., 16 (1985) 186-197.

[25] M. Rahman, A simple evaluation of Askey and Wilson's integral, Proc. Am. Math. Soc. 92 (1984) 413-417.

[26] M. Rahman, An integral representation of a ${ }_{10} \phi_{9}$ and continuous biorthogonal ${ }_{10} \phi_{9}$ rational functions, Canad. J. Math. 38 (1986) 605-618.

[27] P. J. Szablowski, On the structure and probabilistic interpretation of Askey-Wilson densities and polynomials with complex parameters, J. Funct. Anal. 261 (2011) 635-659. 
[28] A. Verma, V. K. Jain, Transformations between basic hypergeometric series on different bases and identities of Rogers-Ramanujan type, J. Math Anal. Appl. 76 (1980) 230-269.

School of Mathematical Sciences and S Shanghai Key Laboratory of PMmP, East China Normal University, 500 Dongchuan Road, Shanghai 200241, P. R. China

E-mail address: zgliu@math.ecnu.edu.cn; liuzg@hotmail.com 